4403

\section{Cure Quest: Team Science of Game Design for Medical} Education

Benjamin Chang ${ }^{1}$, Shawn Lawson ${ }^{2}$, Kathleen Ruiz ${ }^{2}$, Mei Si² ${ }^{2}$ Emilia Bagiella, $\mathrm{PhD}^{3}$, Emma K T Benn, DrPH, MPH${ }^{3}$, and Janice Lynn Gabrilove, MD, FACP ${ }^{3}$

${ }^{1}$ RPI, School of Humanities, Arts and Social Sciences; ${ }^{2}$ Rensselaer Polytechnic Institute; ${ }^{3}$ Mount Sinai School of Medicine

OBJECTIVES/GOALS: "Cure Quest" is an adventure quest game about the process of new drug discovery and development. The player explores a magical island in search of a cure for a mysterious illness, traveling through different lands based on the stages of the drug discovery pipeline. Along the way, they must solve puzzles, decipher clues, and enlist the help of a 'team science' group of collaborators. The game uses a fantastical setting and engaging story to communicate the topic through metaphorical representations, instilling a sense of wonderment in the learning process. Real-world science is embedded into fictionalized lands such as the Labyrinth of Target Identification, the Forest of Small Molecule Discovery, the Tree of Biostatistics, the Mountains of FDA Approval and the Desert of Funding. The project represents a novel application of game-based learning to a complex topic not typically addressed through games. The process of designing and developing the game itself uncovers strong parallels between the interdisciplinary game design process and the interdisciplinary team science process. The objective of the game is to communicate high-level concepts of the drug discovery and development process, starting with the principles of ethical research and the motivations behind medical discovery, through the development of a new drug and finally to FDA approval. The goal is to improve understanding of clinical translational science among the different disciplines involved, and to raise overall awareness of the drug discovery process. METHODS/ STUDY POPULATION: The game is being developed through a collaboration between faculty and students at ISMMS and the Games and Simulation Arts and Science Program at Rensselaer Polytechnic Institute. The first target audience is 2nd-3rd year medical students, with the future goal of adapting the game to a broader population. The game design is informed by specific learning outcomes, input from players in the target population and an ongoing iterative design process. The game is designed for mobile devices (iOS and Android), with an emphasis on narrative, exploration, and puzzle solving. Future evaluation will be performed through a quasi-experimental design comparing standard lectures with the game on a drug discovery. RESULTS/ANTICIPATED RESULTS: The game is currently in development, but the project has yielded insight into the design process for serious games in medicine. We found that for a game of this type it is essential not just to have both designers and subject matter experts, but to enable cross-pollination of modes of thinking. Through multiple design iterations and focus groups, we found that a game design approach rooted in narrative and allegorical abstraction would have a better ability to engage the target audience than one focused only on realistic simulation. When complete, we anticipate that the game will improve understanding of the core concepts in drug discovery. CureQuest is designed as an episodic game, following the sequence of stages in the drug discovery and development process. In this version of the game, we demonstrate five of the initial episodes: The City of Discovery of Unmet Medical Need; The Labyrinth of Target Identification; the Aquarium of Transgenic Phenotype Expression; the Rival Researcher Gang Quiz Battle; and the Desert of Funding.
DISCUSSION/SIGNIFICANCE OF IMPACT: If successful, the game-based learning approach can help fill key gaps in current formal medical and scientific training, as well as gaps in understanding among the general public. The design process serves as an informative model of evolving collaborative team science.

4507

Developing and implementing a Principal Investigator (PI) primer to improve the conduct of human research at the University of Minnesota

Jessica Wright ${ }^{1}$, Jennifer Maas ${ }^{1}$, and Megan Hoffman ${ }^{1}$

${ }^{1}$ University of Minnesota CTSI

\section{OBJECTIVES/GOALS:}

1. Assess the institutional and individual training needs and gaps in the conduct of human research for PIs at the University of Minnesota.

2. Define the training program's learning objectives.

3. Develop and implement an in-person training session that addresses the gaps.

\section{METHODS/STUDY POPULATION:}

- Establish a planning committee

- Identify required and optional training that is already available for PIs, then determine gaps

- Understand research training needs based on conversations with departmental and human research protection program leaders.

- Develop learning objectives and curriculum based on Federal and Local regulations, guidelines, and policies.

- Establish a feedback loop regarding research compliance with the HRPP, to assess trends and ensure continuous improvement.

- Evaluate the training program's participants using confidence and satisfaction measures.

RESULTS/ANTICIPATED RESULTS: Developed and piloted a 90minute in-person training program entitled "PI Primer" with the goals of:

- Increasing awareness and knowledge of the role and responsibilities of the Principal Investigator (PIs) according to the International Harmonization for Good Clinical Practice (ICHGCP), Federal Regulations (FDA, DHHS, ect.), and University of Minnesota Policies.

- Identifying root causes for receiving an FDA 483 (inspection findings).

- Addressing and preventing common inspection findings (CAPA).

- Describe individual and institutional conflict of interest (COI), and identify the key steps necessary to manage COIs.

DISCUSSION/SIGNIFICANCE OF IMPACT: OHRP's guidance on the "Responsibilities of Investigators" states that it is the Institution's responsibility to provide human research training on a wide variety of topics to ensure the ethical conduct of research and protection of participants. PI Primer provides an in-person forum for investigators to build upon required responsible conduct of research and good clinical practice training to be able to apply the role and responsibilities of a PI to their own research. PI Primer also establishes a network of PIs in order to enhance connectivity and shared learning. 